Supplement of Biogeosciences, 17, 715-726, 2020

https://doi.org/10.5194/bg-17-715-2020-supplement

(C) Author(s) 2020. This work is distributed under

the Creative Commons Attribution 4.0 License.

(c) (1)

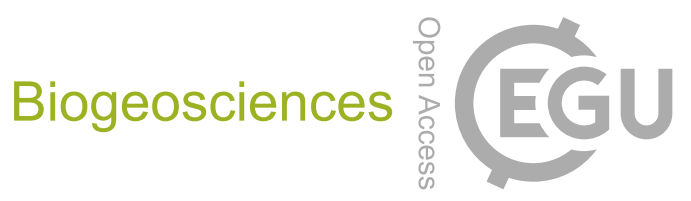

Supplement of

\title{
Increasing soil carbon stocks in eight permanent forest plots in China
}

Jianxiao Zhu et al.

Correspondence to: Jingyun Fang (jyfang@urban.pku.edu.cn)

The copyright of individual parts of the supplement might differ from the CC BY 4.0 License. 


\section{Supporting information}

3 Supplementary Materials and Methods

$4 \quad$ Study sites and field measurements

5 The sites of this study were selected in boreal, temperate, subtropical and tropical forests

6 spanning approximately $26^{\circ}$ latitude in the eastern China (Fig. 1, Table 1). The detailed

7 information about each site is described below.

8 Boreal forest: The boreal site was established in Tahe, Great Xing'anling, northeastern China $9 \quad\left(52^{\circ} 38^{\prime} 42^{\prime \prime} \mathrm{N}, 123^{\circ} 46^{\prime} 08^{\prime \prime} \mathrm{E}\right)$, in May 1998 (Wang et al., 2001). The topography is gently

10 undulating with an average slope of $10^{\circ}$. The elevation is $466 \mathrm{~m}$. The mean annual temperature (MAT) and precipitation (MAP) are $-4.3{ }^{\circ} \mathrm{C}$ and $477 \mathrm{~mm}$, respectively. The frost-free period is shorter than 100 days, and the snow pack lasts for approximately 5 months in this region. The Larix forest was a 100-year-old mature forest at the time of the first sampling, dominated by Larix gmelinii accompanied by Betula platyphylla, Pinus sylvestris, Picea koraiensis, and Populus davidiana. The understory is dominated by Ledum palustre. The parent material is granite bedrock, and the soil is a dark brown forest soil. The soil in the plots has depths of $30-40 \mathrm{~cm}$, with a $\mathrm{pH}$ between 5.0 and 6.0 .

Temperate forests: The temperate site on Mt. Dongling stands near the Xiaolongmen

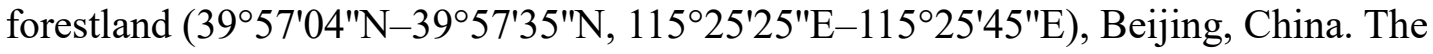
temperate forests in this region are protected and have not experienced serious anthropogenic disturbance (Fang et al., 2007). The MAT and MAP were $4.8^{\circ} \mathrm{C}$ and $612 \mathrm{~mm}$, respectively (Fig. 1, Table 1). We selected three plots from the top to the foot of a mountain as the temperate plots of deciduous broadleaf birch (Betula platyphylla) and oak (Quercus wutaishanica) forests and a pine (Pinus tabuliformis) plantation in 1992. The soil in this region has a depth of $90-110 \mathrm{~cm}$ and a $\mathrm{pH}$ that ranges between 6.0 and 7.0. 
The birch plot is located on a northwest-facing slope near the peak of the mountain, with an elevation of $1,350 \mathrm{~m}$. The forest is dominated by B. platyphylla accompanied by B. utilis and Populus alba. The woody plants in the understory include Sorbus pohuashanensis, Lonicera japonica, Prunus armeniaca, Corylus mandshurica, Acer mono, Abelia biflora, Leptodermis oblonga, Spiraea sargentiana, and Macrocarpium officinalis. The oak plot is located on a southwest-facing slope on the middle of the mountain, with an elevation of 1,150 $\mathrm{m}$. The forest is a secondary forest recovered from human disturbance, dominated by $Q$. wutaishanica accompanied by $B$. utili. The understory woody plants include S. sargentiana, $A$. mono, Lespedeza bicolor, L. japonica, C. mandshurica, and Deutzia scabra. Both the birch and the oak forests are secondary deciduous broadleaf forests (55 years at the time of the first sampling). The pine forest is on a southeast-facing slope at the foot of the mountain, with an altitude of $1,050 \mathrm{~m}$. The pine forest was a 30 -year-old plantation at the time of the first sampling, dominated by only one tree species, P. tabuliformis, with very few plants in the understory and a thick litter floor.

Subtropical forests: The subtropical site is located in the Dinghushan Biosphere Reserve $\left(23^{\circ} 09^{\prime} 21^{\prime \prime} \mathrm{N}-23^{\circ} 11^{\prime} 30^{\prime \prime} \mathrm{N}, 112^{\circ} 30^{\prime} 39^{\prime \prime} \mathrm{E}-112^{\circ} 33^{\prime} 41^{\prime \prime} \mathrm{E}\right)$ in Guangdong Province, China. The region has a typical southern subtropical monsoon climate (warm and humid). The MAP is $1,678 \mathrm{~mm}, 80 \%$ of which falls in the wet season (April to September), and the MAT is $22.3{ }^{\circ} \mathrm{C}$. The altitude in the reserve ranges from $10 \mathrm{~m}$ to $1,000 \mathrm{~m}$. The bedrock is sandstone and shale, with a $\mathrm{pH}$ that ranges between 4.0 and 4.9 .

A $50 \times 50 \mathrm{~m}^{2}$ plot, representative of the monsoon evergreen broadleaf forests in the region, was established in 1979 at an elevation of $275 \mathrm{~m}$ on a south-facing slope. The evergreen broadleaf forest has not been disturbed for more than 400 years (Zhou et al., 2006). The plants in the evergreen plot are typical and natives of tropics and subtropics, including Castanopsis chinensis, Canarium pimela, Schima superba, and Engelhardtia roxburghiana, 
among others. The sub-canopy layer is mainly composed of Cryptocarya concinna and Machilus chinensis. Another two $30 \times 40 \mathrm{~m}^{2}$ plots had also been established in 1979. The pine (Pinus massoniana) plantation and the mature mixed pine and broadleaf forests are the other two most common forest communities that represent the early- and mid-successional stages of monsoon evergreen broadleaf forest, respectively, in this region. The age of the pine plantation was approximately 40 years at the time of the first sampling.

Tropical forest: The tropical site was established in the Jianfengling National Natural Reserve $\left(18^{\circ} 23^{\prime} \mathrm{N}-18^{\circ} 50^{\prime} \mathrm{N}, 108^{\circ} 36^{\prime} \mathrm{E}-109^{\circ} 05^{\prime} \mathrm{E}\right)$ on southwestern Hainan Island, China, in 1992 (Zhou et al., 2013). The region has a typical tropical mountain rain forest with an elevation of $800-1,000 \mathrm{~m}$. The MAT and MAP were $19.8^{\circ} \mathrm{C}$ and $2,449 \mathrm{~mm}$, respectively. The primary forest in this region has not been disturbed for more than 300 years and is dominated by species in families Lauraceae and Fagaceae, e.g., Mallotus hookerianus, Gironniera subaequali, Cryptocarya chinensis, Cyclobalanopsis patelliformis and Nephel-ium topengii. The soils are lateritic yellow soil, with a $\mathrm{pH}$ that ranges between 4.3 and 4.7 .

\section{References}

Fang, J. Y., Liu, G. H., Zhu, B., Wang, X. K., and Liu, S. B.: Carbon budgets of three temperate forest ecosystems in Dongling Mt., Beijing, China. Sci. China Earth Sci., 50, 92-101, https://doi.org/10.1007/s11430-007-2031-3, 2007.

Wang, C., Gower, S. T., Wang, Y., Zhao, H., Yan, P., and Bond-Lamberty, B. P.: The influence of fire on carbon distribution and net primary production of boreal Larix gmelinii forests in north-eastern China. Glob. Change Biol., 7, 719-730, https://doi.org/10.1046/j.1354-1013.2001.00441.x, 2001.

Zhou, G., Liu, S., Li, Z., Zhang, D., Tang, X., Zhou, C., Yan, J., Mo, J.: Old-growth forests can accumulate carbon in soils. Science, 314, 1417, https://doi:10.1126/science.1130168, 

2006.

77 Zhou, Z., Jiang, L., Du, E., Hu, H., Li, Y., Chen, D., and Fang, J.: Temperature and substrate 78 availability regulate soil respiration in the tropical mountain rainforests, Hainan Island, China. J. Plant Ecol., 6, 325-334, https://doi.org/10.1093/jpe/rtt034, 2013. 
81 Table S1. Allometric equations of above-ground biomass by species and sites used in this 82 study. The equations are expressed as $B=\mathrm{a}\left(D^{2} H\right)^{\mathrm{b}}$, where $B, D$, and $H$ are the biomass $(\mathrm{kg})$,

83 DBH $(\mathrm{cm})$ and height $(\mathrm{m})$ of each stem, respectively.

\begin{tabular}{|c|c|c|c|c|c|}
\hline Site & Species & Component & $\mathbf{a}$ & $\mathbf{b}$ & $R^{2}$ \\
\hline \multirow[t]{6}{*}{ Boreal } & \multirow[t]{3}{*}{ Larix gmelinii } & Bole & 0.01258 & 0.99331 & 0.99 \\
\hline & & Branch & 0.00136 & 1.02797 & 0.99 \\
\hline & & Leaf and Fruit & 0.01009 & 0.64543 & 0.98 \\
\hline & \multirow[t]{3}{*}{ Betula platyphylla } & Bole & 0.02853 & 0.89271 & 0.99 \\
\hline & & Branch & 0.00278 & 1.02568 & 0.99 \\
\hline & & Leaf and Fruit & 0.01545 & 0.61265 & 0.98 \\
\hline \multirow[t]{28}{*}{ Temperate } & \multirow[t]{4}{*}{ Pinus tabulaeformis } & Stem & 0.0475 & 0.8539 & 0.98 \\
\hline & & Branch & 0.0017 & 1.1515 & 0.94 \\
\hline & & Leaf & 0.0134 & 0.8099 & 0.92 \\
\hline & & Fruit & 0.0013 & 0.9055 & 0.27 \\
\hline & \multirow[t]{3}{*}{ Betula platyphylla \& B. dahurica } & Stem & 0.0319 & 0.9356 & 0.99 \\
\hline & & Branch & 0.00063 & 1.2781 & 0.91 \\
\hline & & Leaf and Fruit & 0.00016 & 1.1688 & 0.88 \\
\hline & \multirow[t]{3}{*}{ Quercus wutaishanica } & Stem & 0.0369 & 0.9165 & 0.99 \\
\hline & & Branch & 0.00051 & 1.3377 & 0.9 \\
\hline & & Leaf and Fruit & 0.00021 & 1.171 & 0.95 \\
\hline & \multirow[t]{3}{*}{ Populus davidiana } & Stem & 0.2286 & 0.6933 & 0.98 \\
\hline & & Branch & 0.0247 & 0.7378 & 0.96 \\
\hline & & Leaf and Fruit & 0.0108 & 0.8181 & 0.98 \\
\hline & \multirow[t]{3}{*}{ Acer mono } & Stem & 0.03136 & 0.9775 & 0.99 \\
\hline & & Branch & 0.00588 & 1.103 & 0.98 \\
\hline & & Leaf and Fruit & 0.01141 & 0.8803 & 0.98 \\
\hline & \multirow[t]{3}{*}{ Ulmus macrocarpa } & Stem & 0.05229 & 0.891 & 0.99 \\
\hline & & Branch & 0.01233 & 0.9359 & 0.91 \\
\hline & & Leaf and Fruit & 0.01736 & 0.7738 & 0.85 \\
\hline & \multirow[t]{3}{*}{ Fraxinus rhynchophylla } & Stem & 0.06013 & 0.8906 & 0.99 \\
\hline & & Branch & 0.00556 & 1.169 & 0.98 \\
\hline & & Leaf and Fruit & 0.00829 & 0.9919 & 0.98 \\
\hline & \multirow[t]{3}{*}{ Juglans mandshurica } & Stem & 0.02511 & 0.9271 & 0.99 \\
\hline & & Branch & 0.00957 & 0.974 & 0.86 \\
\hline & & Leaf and Fruit & 0.08725 & 0.2634 & 0.81 \\
\hline & \multirow[t]{3}{*}{ Tilia mongolica } & Stem & 0.0811 & 0.7994 & 0.99 \\
\hline & & Branch & 0.05703 & 0.463 & 0.88 \\
\hline & & Leaf and Fruit & 0.001259 & 0.7802 & 0.98 \\
\hline \multirow[t]{3}{*}{ Sub-tropical } & \multirow[t]{3}{*}{ All species } & Stem & 0.0608 & 2.5585 & 0.97 \\
\hline & & Branch & 0.0254 & 2.587 & 0.97 \\
\hline & & Leaf and Fruit & 0.0385 & 2.0739 & 0.97 \\
\hline \multirow[t]{3}{*}{ Tropical } & \multirow[t]{3}{*}{ All species } & Stem & 0.022816 & 0.992674 & 0.98 \\
\hline & & Branch & 0.005915 & 0.999046 & 0.98 \\
\hline & & Leaf and Fruit & 0.005997 & 0.804661 & 0.98 \\
\hline
\end{tabular}


Table S2. Mean soil organic carbon (SOC) content, bulk density, and SOC stock at the 0-10 and 10-20 cm depths in the 1990s and the 2010s at the four forest biomes.

\begin{tabular}{|c|c|c|c|c|c|c|c|}
\hline \multirow[t]{2}{*}{ Biome } & \multirow[t]{2}{*}{ Forest type } & \multicolumn{3}{|c|}{$0-10 \mathrm{~cm}$} & \multicolumn{3}{|c|}{$10-20 \mathrm{~cm}$} \\
\hline & & $1990 \mathrm{~s}$ & $2010 \mathrm{~s}$ & Change rate & $1990 \mathrm{~s}$ & $2010 s$ & Change rate \\
\hline \multicolumn{8}{|c|}{ SOC content ${ }^{*}$} \\
\hline Boreal & Larch & $7.9 \pm 1.4$ & $8.1 \pm 1.2$ & $+0.02 \pm 0.00$ & $1.8 \pm 0.4$ & $1.9 \pm 0.8$ & $+0.01 \pm 0.00$ \\
\hline \multirow[t]{4}{*}{ Temperate } & Birch & $8.8 \pm 4.5$ & $8.7 \pm 1.7$ & $-0.00 \pm 0.00$ & $3.3 \pm 1.3$ & $3.7 \pm 0.3$ & $+0.02 \pm 0.01$ \\
\hline & Oak & $4.3 \pm 0.1$ & $4.8 \pm 0.6$ & $+0.03 \pm 0.00$ & $3.2 \pm 0.0$ & $3.3 \pm 0.9$ & $+0.01 \pm 0.00$ \\
\hline & Pine & $3.1 \pm 0.4$ & $4.3 \pm 1.5$ & $+0.06 \pm 0.02$ & $2.8 \pm 0.1$ & $3.2 \pm 0.7$ & $+0.02 \pm 0.00$ \\
\hline & Mean & $5.4 \pm 3.0$ & $6.0 \pm 2.4$ & $+0.03 \pm 0.03$ & $3.1 \pm 0.3$ & $3.4 \pm 0.3$ & $+0.02 \pm 0.01$ \\
\hline \multirow[t]{4}{*}{ Subtropical } & Evergreen & $2.5 \pm 0.4$ & $3.6 \pm 0.4$ & $+0.05 \pm 0.01$ & $1.3 \pm 0.2$ & $1.7 \pm 0.3$ & $+0.02 \pm 0.00$ \\
\hline & Mixed & $1.8 \pm 0.5$ & $2.5 \pm 0.4$ & $+0.03 \pm 0.01$ & $1.0 \pm 0.1$ & $1.1 \pm 0.3$ & $+0.01 \pm 0.00$ \\
\hline & Pine & $1.1 \pm 0.3$ & $1.7 \pm 0.2$ & $+0.03 \pm 0.01$ & $0.7 \pm 0.2$ & $0.7 \pm 0.2$ & $+0.00 \pm 0.00$ \\
\hline & Mean & $1.8 \pm 0.7$ & $2.6 \pm 1.0$ & $+0.04 \pm 0.01$ & $1.0 \pm 0.3$ & $1.1 \pm 0.5$ & $+0.01 \pm 0.01$ \\
\hline Tropical & Evergreen & $2.5 \pm 0.5$ & $3.2 \pm 1.0$ & $+0.03 \pm 0.01$ & $1.4 \pm 0.2$ & $1.4 \pm 0.3$ & $+0.00 \pm 0.00$ \\
\hline Mean & & $4.0 \pm 2.8$ & $4.6 \pm 2.6$ & $+0.03 \pm 0.02$ & $1.9 \pm 1.1$ & $2.1 \pm 1.2$ & $+0.01 \pm 0.01$ \\
\hline \multicolumn{8}{|c|}{ Bulk density* } \\
\hline Boreal & Larch & $0.3 \pm 0.1$ & $0.3 \pm 0.1$ & $+2.6 \pm 0.5$ & $1.4 \pm 0.3$ & $1.3 \pm 0.2$ & $-5.2 \pm 1.1$ \\
\hline \multirow[t]{4}{*}{ Temperate } & Birch & $0.5 \pm 0.4$ & $0.6 \pm 0.1$ & $+4.6 \pm 2.3$ & $0.9 \pm 0.1$ & $0.9 \pm 0.1$ & $-3.0 \pm 0.3$ \\
\hline & Oak & $0.9 \pm 0.0$ & $0.8 \pm 0.1$ & $-3.6 \pm 0.3$ & $1.0 \pm 0.1$ & $1.0 \pm 0.1$ & $-0.1 \pm 0.0$ \\
\hline & Pine & $1.1 \pm 0.1$ & $0.9 \pm 0.2$ & $-5.5 \pm 0.6$ & $1.1 \pm 0.0$ & $1.1 \pm 0.1$ & $+0.1 \pm 0.0$ \\
\hline & Mean & $0.8 \pm 0.3$ & $0.8 \pm 0.2$ & $-1.5 \pm 5.3$ & $1.0 \pm 0.1$ & $1.0 \pm 0.1$ & $-1.0 \pm 1.7$ \\
\hline \multirow[t]{4}{*}{ Subtropical } & Evergreen & $0.9 \pm 0.1$ & $0.8 \pm 0.0$ & $-3.0 \pm 0.2$ & $1.0 \pm 0.1$ & $0.9 \pm 0.0$ & $-3.4 \pm 0.2$ \\
\hline & Mixed & $1.1 \pm 0.1$ & $0.9 \pm 0.0$ & $-11.3 \pm 0.5$ & $1.1 \pm 0.1$ & $1.1 \pm 0.0$ & $-2.4 \pm 0.1$ \\
\hline & Pine & $1.3 \pm 0.1$ & $1.1 \pm 0.0$ & $-8.5 \pm 0.3$ & $1.3 \pm 0.1$ & $1.1 \pm 0.0$ & $-8.9 \pm 0.3$ \\
\hline & Mean & $1.1 \pm 0.2$ & $0.9 \pm 0.1$ & $-7.7 \pm 4.2$ & $1.1 \pm 0.2$ & $1.0 \pm 0.1$ & $-4.9 \pm 3.5$ \\
\hline Tropical & Evergreen & $1.1 \pm 0.0$ & $1.2 \pm 0.2$ & $+1.9 \pm 0.2$ & $1.1 \pm 0.1$ & $1.2 \pm 0.1$ & $+2.9 \pm 0.2$ \\
\hline Mean & & $0.9 \pm 0.3$ & $0.8 \pm 0.3$ & $-3.1 \pm 5.6$ & $1.1 \pm 0.2$ & $1.1 \pm 0.2$ & $-2.5 \pm 3.6$ \\
\hline \multicolumn{8}{|l|}{ SOC stock } \\
\hline Boreal & Larch & $22.1 \pm 0.9$ & $26.1 \pm 4.9$ & $+247.1 \pm 30.3$ & $25.5 \pm 1.1$ & $25.6 \pm 11.4$ & $+4.0 \pm 0.8$ \\
\hline \multirow[t]{4}{*}{ Temperate } & Birch & $44.2 \pm 1.0$ & $51.8 \pm 2.2$ & $+379.4 \pm 12.6$ & $30.4 \pm 8.8$ & $32.1 \pm 0.8$ & $+82.7 \pm 13.2$ \\
\hline & Oak & $38.6 \pm 2.3$ & $40.1 \pm 10.9$ & $+72.7 \pm 12.3$ & $30.7 \pm 2.6$ & $31.7 \pm 7.5$ & $+47.9 \pm 8.3$ \\
\hline & Pine & $32.5 \pm 2.5$ & $40.7 \pm 9.7$ & $+413.2 \pm 68.4$ & $30.1 \pm 2.6$ & $34.4 \pm 9.5$ & $+217.6 \pm 40.5$ \\
\hline & Mean & $38.4 \pm 5.9$ & $44.2 \pm 6.6$ & $+288.4 \pm 187.5$ & $30.4 \pm 0.3$ & $32.7 \pm 1.5$ & $+116.1 \pm 89.5$ \\
\hline \multirow[t]{4}{*}{ Subtropical } & Evergreen & $22.6 \pm 4.0$ & $30.1 \pm 3.8$ & $+375.0 \pm 55.9$ & $13.1 \pm 2.0$ & $15.5 \pm 3.1$ & $+123.3 \pm 21.6$ \\
\hline & Mixed & $20.1 \pm 5.9$ & $21.9 \pm 3.7$ & $+85.8 \pm 18.8$ & $10.7 \pm 1.5$ & $11.3 \pm 2.8$ & $+31.5 \pm 6.3$ \\
\hline & Pine & $14.1 \pm 3.9$ & $17.9 \pm 2.6$ & $+189.6 \pm 38.0$ & $8.5 \pm 1.9$ & $7.5 \pm 2.0$ & $-51.4 \pm 12.2$ \\
\hline & Mean & $18.9 \pm 4.3$ & $23.3 \pm 6.2$ & $+217.2 \pm 146.6$ & $10.8 \pm 2.3$ & $11.5 \pm 4.0$ & $+34.5 \pm 87.4$ \\
\hline Tropical & Evergreen & $28.5 \pm 6.9$ & $36.5 \pm 6.7$ & $+401.8 \pm 84.1$ & $15.2 \pm 3.9$ & $15.9 \pm 3.6$ & $+39.2 \pm 9.2$ \\
\hline Mean & & $27.8 \pm 10.1$ & $33.1 \pm 11.2$ & $+270.6 \pm 141.5$ & $20.5 \pm 9.6$ & $21.8 \pm 10.5$ & $+61.9 \pm 81.2$ \\
\hline
\end{tabular}

88 "Shown are SOC contents (\%) and their change rates $\left(\% \mathrm{yr}^{-1}\right)$, soil bulk density $\left(\mathrm{g} \mathrm{cm}^{-3}\right)$ and 89 their change rates $\left(\mathrm{mg} \mathrm{cm}^{-3} \mathrm{yr}^{-1}\right)$ and SOC stock $\left(\mathrm{Mg} \mathrm{C} \mathrm{ha}^{-1}\right)$ and their change rates $\left(\mathrm{kg} \mathrm{C} \mathrm{ha}^{-1}\right.$ $90 \mathrm{yr}^{-1}$ ) between the 1990s and the 2010s. 
91 Table S3. Mean soil organic carbon (SOC) content, bulk density, SOC stock and their change rates during the past two decades at eight forest 92 sites, which are categorized into four forest biomes.

\begin{tabular}{|c|c|c|c|c|c|c|c|c|c|c|c|}
\hline & $1990 \mathrm{~s}$ & 2010 s & $\begin{array}{c}\text { Change rate } \\
\left(\% \mathrm{yr}^{-1}\right)\end{array}$ & 1990s & 2010s & \multirow[t]{2}{*}{$\begin{array}{l}\text { Change rate } \\
\left(\mathrm{mg} \mathrm{cm}^{-3} \mathrm{yr}^{-1}\right)\end{array}$} & $1990 \mathrm{~s}$ & $2010 \mathrm{~s}$ & $\begin{array}{c}\text { Change rate } \\
\left(\mathrm{kg} \mathrm{C} \mathrm{ha}^{-1} \mathrm{yr}^{-1}\right)\end{array}$ & $\begin{array}{c}\text { Relative rate } \\
\left(\% \mathrm{yr}^{-1}\right)\end{array}$ \\
\hline \multicolumn{11}{|c|}{$0-20 \mathrm{~cm}$ soil depth } & \\
\hline Boreal & Larch & $2.8 \pm 0.6$ & $3.2 \pm 0.9$ & $+0.02 \pm 0.01$ & $0.9 \pm 0.2$ & $0.8 \pm 0.1$ & $-1.3 \pm 0.3$ & $47.6 \pm 2.0$ & $51.6 \pm 16.3$ & $251.1 \pm 46.4$ & $+0.5 \pm 0.1$ \\
\hline \multirow[t]{4}{*}{ Temperate } & Birch & $5.3 \pm 2.4$ & $5.8 \pm 0.9$ & $+0.03 \pm 0.01$ & $0.7 \pm 0.3$ & $0.7 \pm 0.1$ & $+0.8 \pm 0.2$ & $74.6 \pm 9.8$ & $83.8 \pm 3.0$ & $462.1 \pm 37.2$ & $+0.6 \pm 0.1$ \\
\hline & Oak & $3.7 \pm 0.0$ & $4.0 \pm 0.7$ & $+0.01 \pm 0.00$ & $0.9 \pm 0.1$ & $0.9 \pm 0.1$ & $-1.9 \pm 0.1$ & $69.4 \pm 4.8$ & $71.8 \pm 18.5$ & $120.6 \pm 19.9$ & $+0.2 \pm 0.0$ \\
\hline & Pine & $3.0 \pm 0.3$ & $3.7 \pm 1.1$ & $+0.04 \pm 0.01$ & $1.1 \pm 0.1$ & $1.0 \pm 0.1$ & $-2.7 \pm 0.2$ & $62.5 \pm 5.1$ & $75.1 \pm 19.2$ & $630.8 \pm 111.2$ & $+1.0 \pm 0.2$ \\
\hline & Mean & $4.2 \pm 1.0$ & $4.6 \pm 0.9$ & $+0.03 \pm 0.01$ & $0.9 \pm 0.1$ & $0.9 \pm 0.1$ & $-1.3 \pm 1.8$ & $68.8 \pm 6.1$ & $76.9 \pm 6.2$ & $404.5 \pm 259.9$ & $+0.6 \pm 0.4$ \\
\hline \multirow[t]{4}{*}{ Subtropical } & Evergreen & $1.9 \pm 0.3$ & $2.6 \pm 0.4$ & $+0.04 \pm 0.01$ & $1.0 \pm 0.1$ & $0.9 \pm 0.0$ & $-3.2 \pm 0.2$ & $35.6 \pm 6.0$ & $45.6 \pm 6.9$ & $498.3 \pm 78.8$ & $+1.4 \pm 0.2$ \\
\hline & Mixed & $1.4 \pm 0.3$ & $1.7 \pm 0.3$ & $+0.02 \pm 0.00$ & $1.1 \pm 0.1$ & $1.0 \pm 0.0$ & $-6.9 \pm 0.3$ & $30.8 \pm 7.3$ & $33.3 \pm 6.4$ & $117.3 \pm 25.2$ & $+0.4 \pm 0.1$ \\
\hline & Pine & $0.9 \pm 0.2$ & $1.2 \pm 0.2$ & $+0.01 \pm 0.00$ & $1.3 \pm 0.1$ & $1.1 \pm 0.0$ & $-8.7 \pm 0.3$ & $22.7 \pm 5.8$ & $25.4 \pm 4.5$ & $138.2 \pm 29.7$ & $+0.6 \pm 0.1$ \\
\hline & Mean & $1.4 \pm 0.3$ & $1.8 \pm 0.3$ & $+0.02 \pm 0.01$ & $1.1 \pm 0.1$ & $1.0 \pm 0.0$ & $-6.3 \pm 2.8$ & $29.7 \pm 6.5$ & $34.8 \pm 10.1$ & $251.3 \pm 214.2$ & $+0.9 \pm 0.5$ \\
\hline Tropical & Evergreen & $2.0 \pm 0.4$ & $2.3 \pm 0.7$ & $+0.02 \pm 0.00$ & $1.1 \pm 0.0$ & $1.2 \pm 0.1$ & $+2.4 \pm 0.2$ & $43.6 \pm 10.8$ & $52.5 \pm 10.3$ & $441.0 \pm 96.6$ & $+1.0 \pm 0.2$ \\
\hline Mean & & $2.9 \pm 0.6$ & $3.2 \pm 0.7$ & $+0.02 \pm 0.00$ & $1.0 \pm 0.1$ & $1.0 \pm 0.1$ & $-2.7 \pm 3.7$ & $48.4 \pm 18.8$ & $54.9 \pm 20.6$ & $332.4 \pm 200.2$ & $+0.7 \pm 0.4$ \\
\hline \multicolumn{12}{|c|}{ Whole soil depth } \\
\hline Boreal & Larch & $1.4 \pm 0.2$ & $1.5 \pm 0.1$ & $+0.00 \pm 0.00$ & $1.2 \pm 0.2$ & $1.2 \pm 0.2$ & $+0.8 \pm 0.1$ & $65.6 \pm 11.0$ & $69.4 \pm 6.2$ & $243.4 \pm 31.1$ & $+0.4 \pm 0.1$ \\
\hline \multirow[t]{4}{*}{ Temperate } & Birch & $2.0 \pm 0.3$ & $2.1 \pm 0.2$ & $+0.01 \pm 0.00$ & $1.1 \pm 0.1$ & $1.0 \pm 0.2$ & $-2.8 \pm 0.4$ & $207.0 \pm 31.7$ & $214.8 \pm 19.5$ & $390.8 \pm 47.4$ & $+0.2 \pm 0.0$ \\
\hline & Oak & $2.0 \pm 0.7$ & $2.4 \pm 0.2$ & $+0.02 \pm 0.00$ & $1.2 \pm 0.1$ & $1.0 \pm 0.1$ & $-10.3 \pm 0.9$ & $239.1 \pm 80.4$ & $241.7 \pm 15.2$ & $127.2 \pm 25.3$ & $+0.1 \pm 0.0$ \\
\hline & Pine & $1.8 \pm 0.5$ & $1.9 \pm 0.3$ & $+0.00 \pm 0.00$ & $1.3 \pm 0.1$ & $1.3 \pm 0.1$ & $-0.1 \pm 0.0$ & $231.7 \pm 67.0$ & $238.4 \pm 41.4$ & $332.8 \pm 76.7$ & $+0.1 \pm 0.0$ \\
\hline & Mean & $1.9 \pm 0.1$ & $2.1 \pm 0.1$ & $+0.01 \pm 0.01$ & $1.2 \pm 0.1$ & $1.1 \pm 0.2$ & $-4.3 \pm 5.3$ & $226.0 \pm 16.8$ & $231.6 \pm 14.6$ & $283.6 \pm 138.5$ & $+0.1 \pm 0.1$ \\
\hline \multirow[t]{4}{*}{ Subtropical } & Evergreen & $1.1 \pm 0.1$ & $1.4 \pm 0.1$ & $+0.02 \pm 0.00$ & $1.1 \pm 0.1$ & $1.0 \pm 0.0$ & $-3.6 \pm 0.2$ & $68.4 \pm 5.7$ & $86.6 \pm 4.5$ & $907.5 \pm 60.1$ & $+1.3 \pm 0.1$ \\
\hline & Mixed & $0.7 \pm 0.1$ & $1.0 \pm 0.1$ & $+0.01 \pm 0.00$ & $1.2 \pm 0.1$ & $1.1 \pm 0.04$ & $-3.8 \pm 0.2$ & $51.4 \pm 5.5$ & $67.4 \pm 7.2$ & $763.3 \pm 82.4$ & $+1.5 \pm 0.2$ \\
\hline & Pine & $0.6 \pm 0.1$ & $0.7 \pm 0.1$ & $+0.01 \pm 0.00$ & $1.3 \pm 0.1$ & $1.1 \pm 0.0$ & $-9.0 \pm 0.3$ & $43.5 \pm 5.7$ & $47.7 \pm 6.5$ & $206.6 \pm 28.3$ & $+0.5 \pm 0.1$ \\
\hline & Mean & $0.8 \pm 0.2$ & $1.1 \pm 0.3$ & $+0.02 \pm 0.01$ & $1.2 \pm 0.1$ & $1.1 \pm 0.1$ & $-5.5 \pm 3.0$ & $54.4 \pm 12.7$ & $67.2 \pm 19.5$ & $627.6 \pm 370.1$ & $+1.1 \pm 0.5$ \\
\hline Tropical & Evergreen & $0.7 \pm 0.2$ & $0.8 \pm 0.2$ & $+0.00 \pm 0.00$ & $1.3 \pm 0.0$ & $1.3 \pm 0.1$ & $+0.5 \pm 0.0$ & $94.6 \pm 21.8$ & $102.6 \pm 19.9$ & $397.9 \pm 84.2$ & $+0.4 \pm 0.1$ \\
\hline Mean & & $1.3 \pm 0.3$ & $1.5 \pm 0.2$ & $+0.01 \pm 0.01$ & $1.2 \pm 0.1$ & $1.1 \pm 0.1$ & $-3.5 \pm 4.2$ & $125.2 \pm 85.2$ & $133.6 \pm 83.1$ & $421.2 \pm 274.4$ & $+0.6 \pm 0.5$ \\
\hline
\end{tabular}


Table S4. Measured carbon input rates and ratio of soil accumulation to the above-ground net primary production (ANPP) of the eight forest

95 types.

\begin{tabular}{|c|c|c|c|c|c|c|c|c|}
\hline \multirow[t]{2}{*}{ Parameters } & \multirow{2}{*}{$\begin{array}{l}\text { Boreal } \\
\text { Larch }\end{array}$} & \multicolumn{3}{|l|}{ Temperate } & \multicolumn{3}{|l|}{ Subtropical } & \multirow{2}{*}{$\begin{array}{l}\text { Tropical } \\
\text { Evergreen }\end{array}$} \\
\hline & & Birch & Oak & Pine & Evergreen & Mixed & Pine & \\
\hline AGB & $91.1 \pm 25.0$ & $99.3 \pm 9.0$ & $69.6 \pm 4.4$ & $100.0 \pm 17.4$ & $140.0 \pm 5.5$ & $120.9 \pm 16.3$ & $60.1 \pm 3.4$ & $213.6 \pm 41.4$ \\
\hline Dead wood & $1.3 \pm 0.5$ & $5.6 \pm 0.8$ & $3.3 \pm 0.1$ & $4.5 \pm 0.6$ & $13.2 \pm 0.2$ & $8.7 \pm 5.7$ & $0.1 \pm 0.1$ & $5.7 \pm 0.8$ \\
\hline Soil & $69.4 \pm 6.2$ & $214.8 \pm 19.5$ & $241.7 \pm 15.2$ & $238.4 \pm 41.4$ & $86.6 \pm 7.2$ & $67.4 \pm 6.5$ & $47.7 \pm 4.5$ & $102.6 \pm 19.9$ \\
\hline Ecosystem total & $166.2 \pm 31.7$ & $324.9 \pm 30.3$ & $317.1 \pm 20.2$ & $346.9 \pm 60.2$ & $241.2 \pm 13.3$ & $199.2 \pm 28.8$ & $110.7 \pm 8.5$ & $323.7 \pm 62.3$ \\
\hline AGB growth & $899.4 \pm 411.0$ & $2075.2 \pm 253.3$ & $1209.0 \pm 240.61$ & $2144.4 \pm 495.76$ & $-1000.3 \pm 78.2$ & $1911.0 \pm 207.58$ & $1485.3 \pm 166.9$ & $684.1 \pm 145.0$ \\
\hline Litterfall & $2424.2 \pm 283.1$ & $1630.2 \pm 220.4$ & $1869.8 \pm 249.7$ & $2340.1 \pm 310.0$ & $4160.2 \pm 449.0$ & $4277.3 \pm 272.8$ & $1718.8 \pm 430.0$ & $3970.0 \pm 279.8$ \\
\hline Fallen log & $13.0 \pm 3.7$ & $192.2 \pm 26.0$ & $66.2 \pm 7.4$ & $60.0 \pm 12.8$ & $2070.3 \pm 221.2$ & $679.5 \pm 43.6$ & $210.3 \pm 50.8$ & $1034.3 \pm 71.6$ \\
\hline Standing snag & $3.5 \pm 1.8$ & $337.9 \pm 46.8$ & $343.8 \pm 46.1$ & $148.5 \pm 18.5$ & $346.8 \pm 42.3$ & $76.9 \pm 3.2$ & $236.3 \pm 56.9$ & $803.4 \pm 62.4$ \\
\hline ANPP & $3340.1 \pm 698.8$ & $4235.4 \pm 546.1$ & $3488.8 \pm 544.2$ & $4693.0 \pm 837.5$ & $5577.0 \pm 789.8$ & $6944.7 \pm 528.4$ & $3650.6 \pm 704.7$ & $6491.6 \pm 559.2$ \\
\hline Soil accumulation & $243.4 \pm 31.1$ & $390.8 \pm 47.4$ & $127.2 \pm 25.3$ & $332.8 \pm 76.7$ & $907.5 \pm 60.1$ & $763.3 \pm 82.4$ & $206.6 \pm 28.3$ & $397.9 \pm 84.2$ \\
\hline
\end{tabular}


97 Table S5. Summary for C pools and changes in each component of forests in China over the 98 past two decades.

\begin{tabular}{lcccl}
\hline Component & $\begin{array}{c}\text { Carbon pool } \\
(\mathrm{Pg} \mathrm{C})\end{array}$ & $\begin{array}{c}\text { Carbon density } \\
\left(\mathrm{Mg} \mathrm{ha}^{-1}\right)\end{array}$ & $\begin{array}{c}\text { National sink } \\
\left(\mathrm{Tg} \mathrm{C} \mathrm{yr}^{-1}\right)\end{array}$ & Source \\
\hline Biomass & 6.9 & 41.3 & 70.9 & Guo et al., 2013 \\
Soil & 20.0 & 106.1 & 57.1 & Tang et al., 2018; This study \\
Litter & 0.5 & 3.2 & 2.8 & Zhu et al., 2017 \\
Dead wood & 0.4 & 2.8 & 3.9 & Zhu et al., 2017 \\
Ecosystem & $\mathbf{2 7 . 4}$ & $\mathbf{1 5 3 . 4}$ & $\mathbf{1 3 4 . 7}$ & \\
\hline
\end{tabular}

99 
100 Figure S1. Changes in soil organic carbon contents (left, \%) and bulk densities (right, $\mathrm{g} \mathrm{cm}^{-3}$ ) 101 with soil depth for the eight forests in the 1990s and the 2010s in China. For the details on the 102 sites, see Table 1.

103

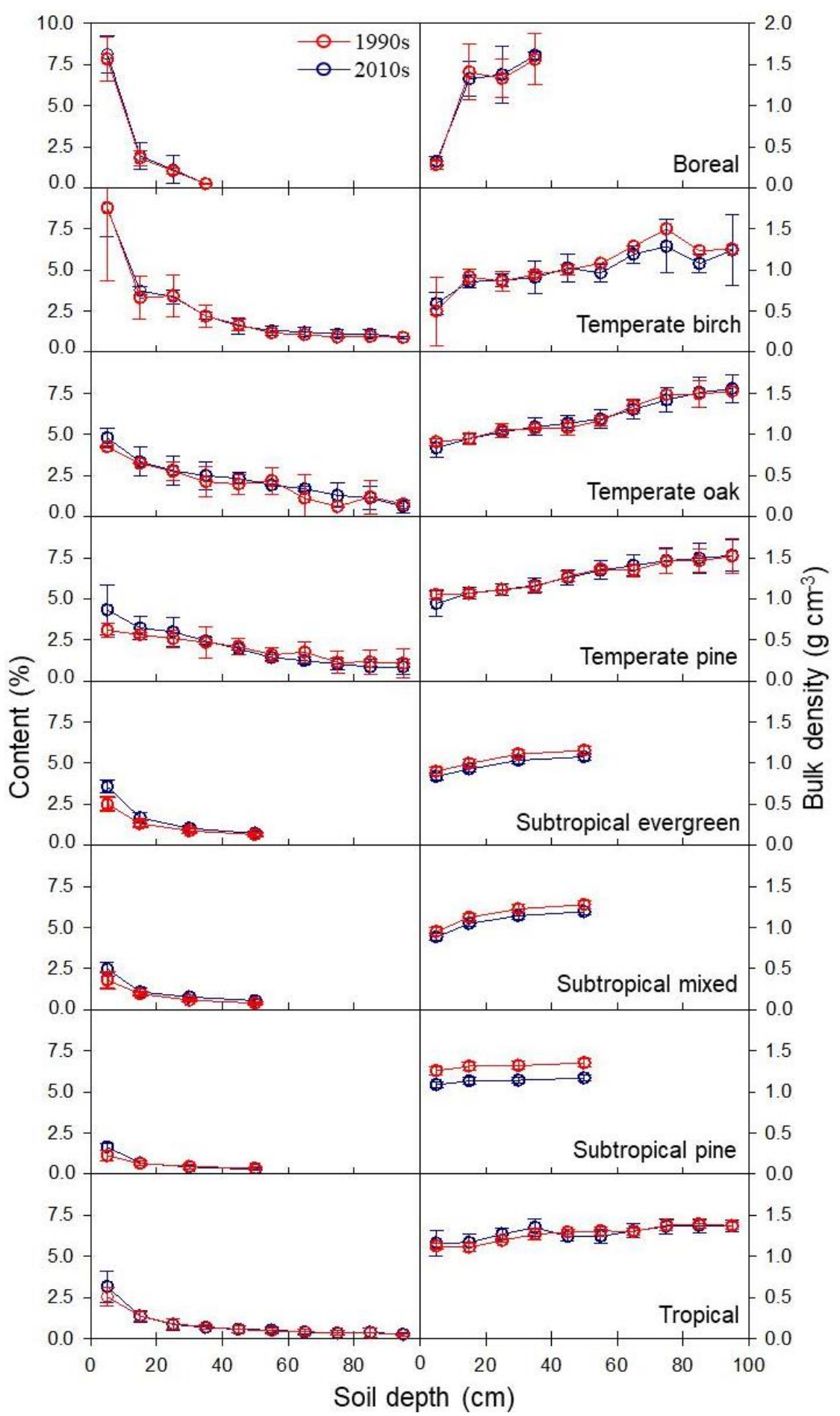


106 Figure S2. Comparison of soil organic carbon stocks of the surface soil depth $(0-20 \mathrm{~cm})$ in 107 the eight forest plots of China between the 1990s and the 2010s. The inset graph shows the 108 SOC change rates of the surface soil depth $(0-20 \mathrm{~cm})$ by forest biomes.

109

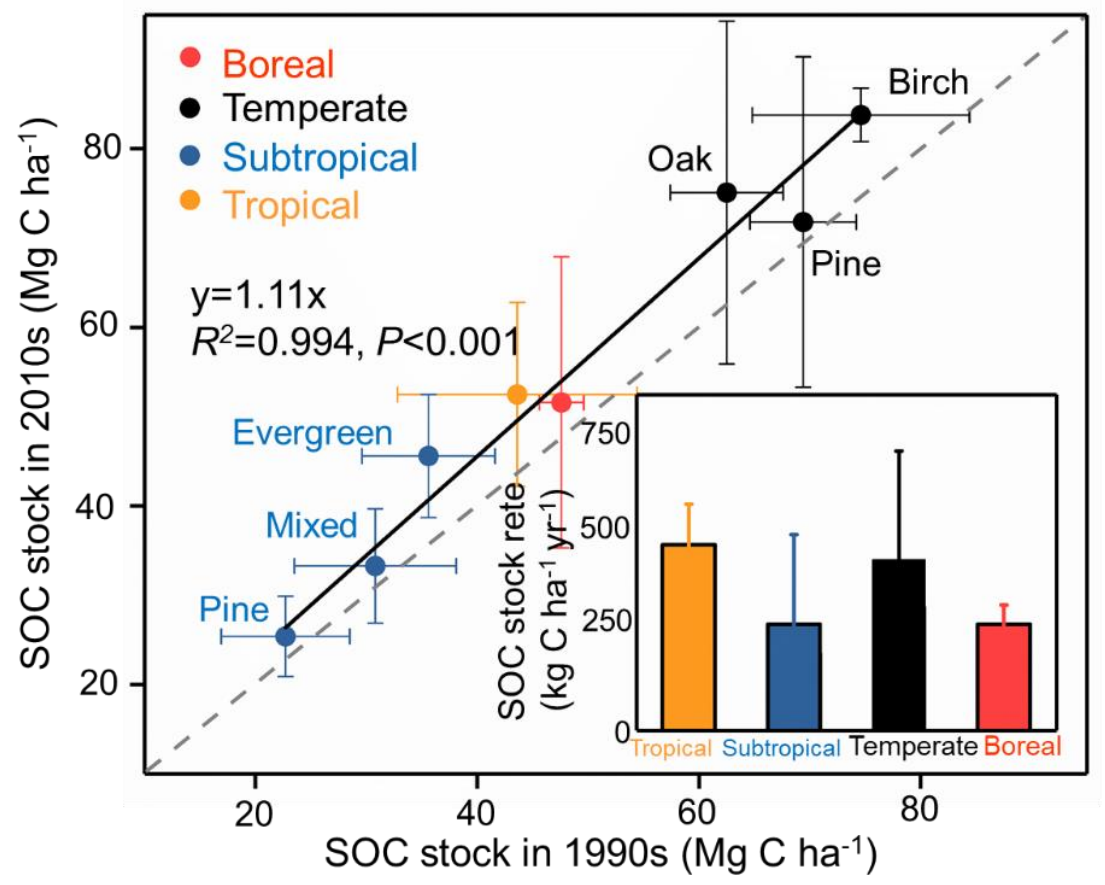

